\title{
The Essence of Principal Typings
}

\author{
J. B. Wells ${ }^{1 \star}$ \\ Heriot-Watt University \\ http://www.cee.hw.ac.uk/ jbw/
}

\begin{abstract}
Let $S$ be some type system. A typing in $S$ for a typable term $M$ is the collection of all of the information other than $M$ which appears in the final judgement of a proof derivation showing that $M$ is typable. For example, suppose there is a derivation in $S$ ending with the judgement $A \vdash M: \tau$ meaning that $M$ has result type $\tau$ when assuming the types of free variables are given by $A$. Then $(A, \tau)$ is a typing for $M$.

A principal typing in $S$ for a term $M$ is a typing for $M$ which somehow represents all other possible typings in $S$ for $M$. It is important not to confuse this notion with the weaker notion of principal type often mentioned in connection with the Hindley/Milner type system. Previous definitions of principal typings for specific type systems have involved various syntactic operations on typings such as substitution of types for type variables, expansion, lifting, etc.

This paper presents a new general definition of principal typings which does not depend on the details of any particular type system. This paper shows that the new general definition correctly generalizes previous system-dependent definitions. This paper explains why the new definition is the right one. Furthermore, the new definition is used to prove that certain polymorphic type systems using $\forall$-quantifiers, namely System F and the Hindley/Milner system, do not have principal typings.
\end{abstract}

\section{Introduction}

\subsection{Background and Motivation}

Why Principal Typings? A term represents a fragment of a program or other system represented in some calculus. In this paper, the examples will be drawn from the $\lambda$-calculus, but much of the discussion can be seen as independent of it. A typing $t$ for a term in a specific type system for a calculus is principal if and only if all other typings for the same term can be derived from $t$ by some set of semantically sensible operations. It is important not to confuse the principal typings property of a type system with the principal types property often discussed in the context of the Hindley/Milner system and the ML programming

\footnotetext{
* This work was partly supported by NSF grants CCR 9988529 and EIA 9806745, EPSRC grant GR/L 36963, and Sun Microsystems equipment grant EDUD-7826990410-US.
} 
language. As described by T. Jim [15]:

\section{Principal Types}

Given: a term $M$ typable with type assumptions $A$.

There exists: a type $\sigma$ representing all possible types for $M$ in $A$.

\section{Principal Typings}

Given: a typable term $M$.

There exists: a judgement $A \vdash M: \tau$ representing all possible typings for $M$.

Principal typings allow compositional type inference, where the procedure of finding types for a term uses only the analysis results for its immediate subfragments, which can be analyzed independently in any order. Compositionality helps with such things as performing separate analysis of program modules (and hence helps with separate compilation) and also helps in making a complete/terminating type inference algorithm. For a system lacking principal typings, any type inference algorithm must either be incomplete (i.e., sometimes not finding a typing even though one exists), be noncompositional, or use something other than typings of the system to represent intermediate results. An example of a noncompositional type inference algorithm is the $\mathcal{W}$ algorithm of Damas and Milner [7] for the Hindley/Milner (HM) type system which is used in programming languages like Haskell and Standard ML (SML). For an SML program fragment of the form (let val $\mathrm{x}=e_{1}$ in $e_{2}$ end), the $\mathcal{W}$ algorithm first analyzes $e_{1}$ and then uses the result while analyzing $e_{2}$.

Why Automated Type Inference? Principal typings help with automated type inference in general. For higher-order languages, the necessary types can become quite complex and requiring all of the types to be supplied in advance is burdensome. It is desirable to have as much implicit typing as possible, where types are omitted by humans who write terms. With type inference, the compiler takes an untyped or partially typed term, and it either completes the typing of the term or reports an error if the term is untypable.

The most widely used type inference algorithm is algorithm $\mathcal{W}$ for HM. The HM system supports polymorphism with quite restricted uses of $\forall$ quantifiers. In practice, the limitations of the HM system on polymorphic types make some kinds of code reuse more difficult [21]. As a result, programmers are often forced into contortions to provide code for which the compiler can find typings. This has motivated a long search for more flexible type systems with good type inference algorithms. Popular candidates have been extensions of the HM system such as System $\mathrm{F}[8,28], \mathrm{F}_{\leq}, \mathrm{F}+\eta$, or $\mathrm{F}_{\omega}$.

In this search, there have been a great number of negative results. For quite some time it seemed that the HM system was as good as a system could get and still have a complete/terminating type inference algorithm. Indeed, for many systems $\left(\mathrm{F}, \mathrm{F}_{\omega}\right.$, etc.), typability (whether an untyped program fragment can be given a type) has been proven undecidable, which means no type inference algorithm can be both complete and terminating for all program fragments. 
Wells proved this for System $\mathrm{F}[36,39]$, the finite-rank restrictions of $\mathrm{F}$ above 3 [18], and $\mathrm{F}+\eta$ [37]. Urzyczyn proved it for $\mathrm{F}_{\omega}$ [33], Pottinger proved it for unrestricted intersection types [27], and Pierce proved that even the subtyping relation of $\mathrm{F}_{\leq}$is undecidable [25]. Even worse, for System $\mathrm{F}$ it seems hard to find an amount of type information less than total that is enough to obtain a terminating type inference algorithm [31].

Along the way, there have been a few positive results, some extensions of the HM system, but, perhaps more interestingly, some with restricted intersection types (cf. recent work on intersection types of arbitrarily high finite ranks by Kfoury, Mairson, Turbak, and Wells [19, 17]).

A New Principal Typing Definition vs. $\forall$ Quantifiers For many years it was not known whether type systems with $\forall$ quantifiers could have principal typings. The first difficulty is simply in finding a sufficiently general systemindependent definition of principal typings. The first such definition is given in this paper. A typing $t$ is defined to be a pair $(A \vdash \tau)$ of a set $A$ of type assumptions together with a result type $\tau$. The meaning $\operatorname{Terms}(t)$ of a typing $t=(A \vdash \tau)$ in a particular type system is defined to be the set of all the program fragments $M$ such that $A \vdash M: \tau$ is provable in the system (meaning " $M$ is well typed with result type $\tau$ under the type assumptions $A$ "). A typing $t_{1}$ is defined to be stronger than typing $t_{2}$ if and only if $\operatorname{Terms}\left(t_{1}\right) \subset \operatorname{Terms}\left(t_{2}\right)$. This is "stronger" because $t_{1}$ is a stronger predicate on terms than $t_{2}$ and provides more information about $M \in \operatorname{Terms}\left(t_{1}\right)$, allowing $M$ to be used in more contexts. (The phrase "more general" is often used in the literature instead of "stronger", but "stronger" seems to cause less confusion.) A typing $t$ is defined to be principal in some system for program fragment $M$ if and only if $t$ is at least as strong as all other typings for $M$ in that system.

Comparison with prior notions of principal typing for various type systems reveals that this new definition either exactly matches the old definitions, or is slightly more liberal and admits some additional principal typings of some program fragments. The new definition seems to be the best system-independent definition that can be obtained.

An important benefit of the new definition is that it can be used to show that various systems with $\forall$ quantifiers do not have principal typings. Using this definition, a proof is given here that System F does not have principal typings. Because the definition used here is liberal, the failure of System F to have principal typings by this definition can be taken to mean that there is no reasonable definition of "principal typings" such that System F has them. A similar proof is presented here for the HM system. The proof for System F can be adapted for related systems such as F $+\eta$, and System F's finite-rank restrictions $\Lambda_{k}$ for $k \geq 3$.

If polymorphism is to be based only on $\forall$ quantifiers, it is not clear how to design a type system with the principal typings property. Even systems with extremely restricted uses of $\forall$ quantifiers such as the HM system do not have principal typings. The lack of principal typings has manifested itself as a diffi- 
culty in making type systems more flexible than the restrictive HM system that also have convenient type inference algorithms. But this difficulty appears to have been due to the use of the $\forall$ quantifier for supporting type polymorphism, because many type systems with intersection types have principal typings.

\subsection{Summary of this Paper's Contributions}

1. An explanation is given of the motivations behind the notion of "principal typing" and how this has affected type inference in widely used type systems such as the simply typed $\lambda$-calculus and HM.

2. A new, system-independent definition of principal typings is presented. It is shown that this definition correctly generalizes existing definitions.

3. The new definition is used to finally prove that HM and System F do not have principal typings. Proving this was impossible before due to the lack of a system-independent definition.

\section{Definitions}

This paper restricts attention to the pure $\lambda$-calculus extended with one constant c of ground type. The notation generally follows [2].

The common features of the type systems discussed in this paper are as follows. The type systems considered are restricted to those deriving judgements of the form $A \vdash M: \tau$. There are many interesting type systems that derive judgements with more information, e.g., effect systems deriving judgements of the form $A \vdash M: \tau, \phi$ where $\phi$ is information about the side effects of evaluating $M[16,32]$. To extend the machinery here to such type systems, the extra information should be considered part of the typing which is defined below.

Types Each type system $S$ will have a set of types $\operatorname{Types}(S)$. Let $\sigma, \tau$, and $\rho$ range over types. Included in the set of types are an infinite set of type variables, ranged over by $\alpha, \beta$, and $\gamma$. There will also be one ground type, o, added to help illustrate certain typing issues. For a type system $S$ considered in this paper, the set Types $(S)$ will be some subset of the set Types of types given by the following pseudo-grammar:

$$
\sigma, \tau, \rho::=\alpha|\circ|(\sigma \rightarrow \tau)|(\sigma \cap \tau)|(\forall \alpha \tau)
$$

The free type variables $\operatorname{FTV}(\tau)$ of the type $\tau$ are those variables not bound by $\forall$. Types are identified which differ only by $\alpha$-conversion. Let $s$ range over type substitutions, finite maps from a set of type variables to types.

Typings A pair $x: \sigma$ is a type assumption. A finite set of type assumptions is a type environment. Let $A$ range over type environments. In this paper, type environments are required to mention each term variable at most once. Thus, a 
type environment may be seen as a finite map from a set of term variables to types. Let $A(x)$ be undefined if $x$ is not mentioned in $A$ and otherwise be the unique type $\tau$ such that $(x: \tau) \in A$. Let $A_{x}=\{y: \tau \mid(y: \tau) \in A$ and $y \neq x\}$. Let $\operatorname{FTV}(A)=\bigcup_{(x: \tau) \in A} \operatorname{FTV}(\tau)$. Let $s(A)=\{(x: s(\tau)) \mid(x: \tau) \in A\}$.

In this paper, a typing judgement is a triple of a type environment $A$, a $\lambda$-term $M$, and a result type $\tau$ with the meaning " $M$ is well typed with result type $\tau$ under the type assumptions $A$ ". Rather than the traditional notation $A \vdash M: \tau$, this paper instead writes judgements in the form $M:(A \vdash \tau)$. Although this slight change in notation will simplify the notation later, its real importance is that the new perspective it provides will help dispell widespread misunderstanding of principal typings in the research community. The pair $A \vdash \tau$ is called a typing. Let $t$ range over typings. If $t=(A \vdash \tau)$, let $\mathrm{FTV}(t)=\mathrm{FTV}(A) \cup$ $\operatorname{FTV}(\tau)$ and let $s(t)=(s(A) \vdash s(\tau))$.

For a type system $S$, let the statement $S \triangleright M: t$ hold iff the judgement $M: t$ is derivable using the typing rules of $S$. A type system $S$ assigns typing $t$ to term $M$ iff $S \triangleright M: t$. Let Terms ${ }_{S}(t)=\{M \mid S \triangleright M: t\}$ and let Typings ${ }_{S}(M)=$ $\{t \mid S \triangleright M: t\}$.

Ordering Typings This paper introduces a new ordering on typings in a type system $S$ according to how much information they provide about terms to which they can be assigned, with typings that are lower in the order providing more information. Let $t_{1} \leq_{S} t_{2}$ iff $\operatorname{Terms}_{S}\left(t_{1}\right) \subseteq \operatorname{Terms}_{S}\left(t_{2}\right)$.

Remark 1. Suppose $t_{1} \leq_{S} t_{2}$. Then typing $t_{1}$ can be viewed as providing more information about any $M \in \operatorname{Terms}\left(t_{1}\right)$. If $t_{1}$ and $t_{2}$ are viewed as predicates on terms, then $t_{1}(M)$ implies $t_{2}(M)$ for any $M$. In practice, if all that is known about a term is whether $t_{1}$ or $t_{2}$ can be assigned to it, then knowing that $M \in \operatorname{Terms}\left(t_{1}\right)$ represents an increase in knowledge over knowing that $M \in \operatorname{Terms}\left(t_{2}\right)$. This increased knowledge enlarges the set of contexts in which it is known that it is safe to use $M$.

Remark 2. It is necessary to point out that in the case of the system $F$ (defined below), the relation $\leq_{\mathrm{F}}$ is very different from type containment relations that have been defined in the literature. For example, in [23] Mitchell defines two relations denoted by the symbol $\subseteq$, which will be referred to here as $\subseteq_{1}$ and $\subseteq_{2}$. The relations $\subseteq_{1}$ and $\subseteq_{2}$ are defined for types rather than typings, so they can not possibly provide a definition of principal typings as they are. They must be extended to typings in order to even make a comparison. The only way to do this that even comes close to making any sense is to define $t_{1} \subseteq_{i} t_{2}$ iff $t_{j}=\left(\left\{x_{1}: \tau_{j, 1}, \ldots, x_{n}: \tau_{j, n}\right\} \vdash \tau_{j}\right)$ and $\sigma_{j}=\forall .\left\{f_{1}: \tau_{j, 1}, \ldots, f_{n}: \tau_{j, n}\right\} \rightarrow \tau_{j}$ for $j \in\{1,2\}$ and $\sigma_{1} \subseteq_{i} \sigma_{2}$, where $\forall . \tau$ means to quantify all free type variables in $\tau$. Of course, $\subseteq_{1}$ and $\subseteq_{2}$ must first be extended to handle record subtyping in the obvious way. Given this definition, it turns out that we can exhibit a pair $\left(t_{1}, t_{2}\right) \in\left(\leq_{\mathrm{F}} \backslash \subseteq_{1}\right)$ and a pair $\left(t_{3}, t_{4}\right) \in\left(\subseteq_{2} \backslash \leq_{\mathrm{F}}\right)$, as follows:

$$
\begin{array}{ll}
t_{1}=(\{x: \beta\} \vdash \forall \alpha \alpha) & t_{2}=(\{x: \beta\} \vdash \gamma) \\
t_{3}=(\{x: \beta\} \vdash \gamma \rightarrow \gamma) & t_{4}=(\{x: \beta\} \vdash(\forall \alpha \alpha) \rightarrow \gamma)
\end{array}
$$


In my opinion, the difference is caused by the fact that both relations $\subseteq_{1}$ and $\subseteq_{2}$ are defined w.r.t. a model in which obtaining the meaning of a term requires first assigning meanings to all free variables. This second-class treatment of free variables is not truly compatible with obtaining principal typings.

Some Specific Type Systems The formulation of the simply typed lambda calculus (STLC) presented here is in Curry style, meaning that type information is assigned to pure $\lambda$-terms [6]. The set Types(STLC) is the subset of Types containing all types which do not mention $\forall$ or $\cap$. The typing rules of STLC are CON, VAR, APP, and ABS:

$$
\begin{array}{llll}
\text { CON } & & \Rightarrow \mathrm{c}:(A \vdash \mathrm{o}) \\
\text { VAR } & A(x)=\tau & \Rightarrow & x:(A \vdash \tau) \\
\text { APP } \quad(M:(A \vdash \sigma \rightarrow \tau) \text { and } N:(A \vdash \sigma)) & \Rightarrow & (M N):(A \vdash \tau) \\
\text { ABS } & M:\left(A_{x} \cup\{x: \sigma\} \vdash \tau\right) & \Rightarrow & (\lambda x . M):(A \vdash \sigma \rightarrow \tau)
\end{array}
$$

The Hindley/Milner type system (HM) is an extension of STLC which was introduced by Milner for use in the ML programming language [21, 22]. The HM system introduces a syntactic form (let $x=M$ in $N$ ) for allowing definitions with polymorphic types. The set Types(HM) is the subset of Types containing all types which do not mention $\cap$ and which do not mention $\forall$ inside either argument of a function type constructor (" $\rightarrow$ "). In a typing $(A \vdash \tau)$, the type $\tau$ must not mention $\forall$. The typing rules of HM are CON, APP, and ABS from STLC and the new typing rules $\mathrm{VAR}_{\mathrm{HM}}$ and LET:

$$
\begin{array}{cc}
\operatorname{VAR}_{\mathrm{HM}} & (A(x)=\forall \vec{\alpha} \cdot \tau \text { and } \operatorname{dom}(s)=\{\vec{\alpha}\}) \Rightarrow x:(A \vdash s(\tau)) \\
& \left(M:(A \vdash \tau) \text { and }\{\vec{\alpha}\}=\operatorname{FTV}(\tau) \backslash \mathrm{FTV}(A) \text { and } N:\left(A_{x} \cup\{x: \forall \vec{\alpha} . \tau\} \vdash \sigma\right)\right) \\
\text { LET } & \Rightarrow(\text { let } x=M \text { in } N):(A \vdash \sigma)
\end{array}
$$

Girard formulated System F [8] (independently invented by Reynolds [28]) in the Church style, with explicitly typed terms. The Curry style presentation of $F$ which is given here was first published by Leivant [20]. The set Types $(F)$ is the subset of Types containing all types which do not mention $\cap$. The typing rules of $F$ are CON, VAR, APP, and ABS from STLC and the new typing rules INST and GEN:

$$
\begin{array}{llll}
\text { INST } & (M:(A \vdash \forall \alpha \sigma) \text { and } s=\{\alpha \mapsto \tau\}) & \Rightarrow & M:(A \vdash s(\sigma)) \\
\text { GEN } & (M:(A \vdash \sigma) \text { and } \alpha \notin \operatorname{FTV}(A)) & \Rightarrow & M:(A \vdash \forall \alpha \sigma)
\end{array}
$$

\section{$3 \quad$ History of Principal Typings}

\subsection{Basic Motivations and STLC}

The notions of principal type and principal typing (which has also been called principal pair) first occurred in the context of type assignment systems for the 
$\lambda$-calculus or combinatory logic using simple types. The motivation was determining whether a term is typable and finding types for the term if it is typable. The key idea is to define the typing algorithm by structural recursion on terms. This means that in calculating types for a term $M$, the algorithm will invoke itself recursively on the immediate subterms of $M$. For this to work, the result returned by the recursive invocations must be sufficiently informative.

Example 1. This example illustrates why there is a need for some sort of "most general" typing in the process of inferring type information. Consider these $\lambda$ terms:

$$
M=\lambda z \cdot N P \quad N=\lambda w \cdot w(w z) \quad P=\lambda y x \cdot x
$$

A type inference algorithm Inf for STLC defined using structural recursion would generate a call tree like this:

$$
\operatorname{lnf}(M)=\mathrm{Cmb}_{\lambda}(z, \operatorname{Inf}(N P))=\mathrm{Cmb}_{\lambda}\left(z, \mathrm{Cmb}_{@}(\operatorname{Inf}(N), \operatorname{Inf}(P))\right)
$$

Here the algorithm Inf uses two subalgorithms $\mathrm{Cmb}_{\lambda}$ and $\mathrm{Cmb}$ @ to combine the results from recursively processing the subterms.

Suppose the recursive call to $\ln f(P)$ were to return the typing $t_{1}=(\emptyset \vdash$ $\alpha \rightarrow \alpha \rightarrow \alpha$ ), which is a derivable STLC typing for $P$. Unfortunately, there would be no way that $\mathrm{Cmb}_{@}$ could combine this result with any typing for $N$ to yield a typing for $N P$. The application $(w(w z))$ inside $N$ needs $w$ to have a type of the shape $\sigma \rightarrow \sigma$ for some $\sigma$. This could be solved by using a typing like $t_{2}=(\emptyset \vdash(\alpha \rightarrow \alpha) \rightarrow \alpha \rightarrow \alpha)$ for $P$.

However, the only thing that $\mathrm{Cmb}_{0}$ knows about the subterm $P$ is the typing $t_{1}$, which does not imply the typing $t_{2}$. This can be seen from the following example of a term $P^{\prime} \in \operatorname{Terms}\left(t_{1}\right) \backslash \operatorname{Terms}\left(t_{2}\right)$ :

$$
P^{\prime}=\lambda x y \cdot(\lambda z \cdot x)(\lambda w \cdot w x(w y x))
$$

To see more precisely why $P^{\prime} \in \operatorname{Terms}\left(t_{1}\right)$, here is a type-annotated version:

$$
P^{\prime}=\lambda x^{\alpha} \cdot \lambda y^{\alpha} \cdot\left(\lambda z^{(\alpha \rightarrow \alpha \rightarrow \alpha) \rightarrow \alpha} \cdot x\right)\left(\lambda w^{\alpha \rightarrow \alpha \rightarrow \alpha} \cdot w x(w y x)\right)
$$

It should also be clear why $P^{\prime} \notin \operatorname{Terms}\left(t_{2}\right)$ - the types of $x$ and $y$ are forced to be the same by the applications $(w x)$ and $(w y)$, and this prevents $P^{\prime}$ from having a result type of the shape $\sigma \rightarrow \sigma$. Thus, $t_{1} \not \leq t_{2}$.

The problem here is that the result that $\operatorname{lnf}(P)$ returned is not the most general result. It would have been more useful to have returned the result $t_{3}=$ $(\emptyset \vdash \beta \rightarrow \alpha \rightarrow \alpha)$. In fact, $t_{3}$ is in a certain sense the best possible result, because it can be checked that $t_{3} \leq t$ for every $t \in \operatorname{Typings}(P)$.

To avoid the kind of problems mentioned in example 1, type inference algorithms have been designed so that their intermediate results for subterms are, in some sense, most general. There have been several different ways of characterizing the needed notions of "most general". Hindley [10] gives the following definitions which were intended for use with STLC. 
Definition 1 (Hindley's Principal Type). A principal type in system $S$ of a term $M$ is a type $\tau$ such that

1. there exists a type environment $A$ such that $S \triangleright M:(A \vdash \tau)$, and

2. if $S \triangleright M:\left(A^{\prime} \vdash \tau^{\prime}\right)$, then there exists some $s$ such that $\tau^{\prime}=s(\tau)$.

Hindley used the name "principal pair" for what is called here "principal typing".

Definition 2 (Hindley's Principal Typing). A principal typing in system $S$ of a term $M$ is a typing $t=(A \vdash \tau)$ such that

1. $S \triangleright M: t$, and

2. if $S \triangleright M: t^{\prime}$ for some typing $t^{\prime}=\left(A^{\prime} \vdash \tau^{\prime}\right)$, then there exists some $s$ such that $A^{\prime} \supseteq s(A)$ and $\tau^{\prime}=s(\tau)$.

Clearly, if $t=(A \vdash \tau)$ is a principal typing for a term $M$, then the result type $\tau$ is a principal type. The key property satisfied by STLC w.r.t. these definitions is the following. (See [10] for the history of this discovery.)

Theorem 1 (Principality for STLC). Every term typable in STLC has a principal typing and a principal type. Also, there is an algorithm that decides if a term is typable in STLC and if the answer is "yes" outputs the principal typing.

Hindley gave a further definition of a principal derivation (called by Hindley "deduction") which is not needed for this discussion. These definitions of Hindley essentially represent the earlier approaches of Curry and Feys [6] and Morris [24]. There are two important aspects of this approach:

1. The notion of "more general" is tied to substitution and weakening. For STLC, this exactly captures what is needed, but this fails for more sophisticated type systems.

2. The literature using these definitions freely switches between "principal type" and "principal typing" (or "principal pair"). The algorithms for STLC which are described as having the goal of calculating the "principal type" in fact are designed to calculate principal typings. Because for STLC every term has both a principal typing and a principal type, many people did not pay much attention to the difference. For more sophisticated type systems the difference becomes important.

\subsection{Type Polymorphism and HM}

Although STLC is well behaved, in practice it is quite insufficient for programming languages. To overcome the limitations of STLC, various approaches to adding type polymorphism have been explored and for each approach efforts have been directed to the problem of type inference.

One approach to adding type polymorphism is System F, which was discovered around the beginning of the early 1970s. Towards the end of that decade people were thinking about Curry-style presentations of $\mathrm{F}$ and how to perform type inference for it [20]. In the mid-1990s, I proved that typability for $F$ is undecidable and that therefore there is no complete and always terminating type 
inference algorithm for $F[36,39]$. Later in this paper, the further result that $F$ does not have principal typings is proven.

So far, the most successful and widely used approach to adding type polymorphism is the Hindley/Milner (HM) system, an extension of STLC and also a restriction of $F$. The approach to type inference for $\mathrm{HM}$ differs from that for STLC, because Hindley's notion of principal typing (needed by the type inference algorithms used for STLC) quite clearly does not hold for HM.

Example 2. This example illustrates why definition 2 is not useful for HM. Consider the $\lambda$-terms $M=($ let $x=(\lambda y . y)$ in $N)$ and $N=(x x)$. The term $M$ is typable in HM. For example, the judgement $M: t$ where $t=(\emptyset \vdash \alpha \rightarrow \alpha)$ can be derived in HM. A derivation of this typing might use as an intermediate step the assignment of the typing $t_{1}=(\{x: \forall \beta . \beta \rightarrow \beta\} \vdash \alpha \rightarrow \alpha)$ to the subterm $N$. Given any $\sigma$, let $\sigma^{0}$ stand for $\sigma$ and let $\sigma^{i+1}$ stand for $\sigma^{i} \rightarrow \sigma^{i}$. Thus, using the new abbreviation, $t_{1}=\left(\left\{x: \forall \beta \cdot \beta^{1}\right\} \vdash \alpha^{1}\right)$. The subterm $N$ can in fact be assigned for any $i \geq 0$ the typing $t_{i}=\left(\left\{x: \forall \beta . \beta^{i}\right\} \vdash \alpha^{i}\right)$. And for distinct $i, j \geq 0$, there is no substitution $s$ such that $t_{i}=s\left(t_{j}\right)$. Furthermore, it is not hard to check for $i \geq 0$ that for any other typing $t^{\prime}$ assignable to $N$, that there is no substitution $s$ such that $t_{i}=s\left(t^{\prime}\right)$. So $N$ has no principal typing using definition 2 . In contrast, the term $M$ does have a principal typing by that definition, namely $t$. Although some HM-typable terms (e.g., $N$ ) have no principal typings by definition 2 , it turns out that any HM-typable term with no free variables does.

Until this paper, it was not known whether we were simply not clever enough to conceive of a set of operations which would yield all other HM typings from (hypothetical) HM principal typings. Later in this paper, it is shown that there is no reasonable replacement definition of principal typing that will work for HM.

Milner's cleverness was in finding a way around this problem. The key lies in in the following definition (a clear statement of which can be found in [9]).

Definition 3 ( $A$-Typable and $A$-Principal).

1. A term $M$ is A-typable in $\mathrm{HM}$ iff there is some $A^{\prime}$ mentioning only monotypes (types without any occurrence of " $\forall$ ") and some type $\tau$ such that $M:\left(A \cup A^{\prime} \vdash\right.$

$\tau)$ is derivable in $\mathrm{HM}$.

2. A typing $\left(A \cup A^{\prime} \vdash \tau\right)$ is $A$-principal for term $M$ in $\mathrm{HM}$ iff

(a) $A^{\prime}$ mentions only monotypes,

(b) $M:\left(A \cup A^{\prime} \vdash \tau\right)$ is derivable in $\mathrm{HM}$, and

(c) whenever $M:\left((s(A)) \cup A^{\prime \prime} \vdash \tau^{\prime}\right)$ for $A^{\prime \prime}$ mentioning only monotypes is derivable, there is a substitution $s^{\prime}$ which behaves the same as $s$ on $\operatorname{FTV}(A)$ such that $A^{\prime \prime} \supseteq\left(s^{\prime}\left(A^{\prime}\right)\right)$ and $\tau^{\prime}=s^{\prime}(\tau)$.

The property that HM satisfies w.r.t. the above definition is the following, due to Damas and Milner [7].

Theorem 2 (Principality for $\mathrm{HM}$ ). If a term is A-typable in $\mathrm{HM}$, then it has a A-principal typing in $\mathrm{HM}$. Also, there is an algorithm that decides if a term is A-typable in $\mathrm{HM}$ and if the answer is "yes" outputs its A-principal typing. 
It is not hard to see that a closed term (with no free variables) is $A$-typable iff it is $\emptyset$-typable. So theorem 2 implies that typability is decidable for closed programs and that closed programs have $\emptyset$-principal typings. This is good enough for use in a type inference algorithm for a programming language implementation and, indeed, the HM type system has been very widely used as a result. There are some drawbacks that should be noticed:

1. In order to take advantage of the notion of $A$-principality, any polymorphic types to be used in a term $M$ must be determined before analyzing $M$. So in analyzing a subterm of the shape (let $x=N$ in $M$ ), the subterm $N$ must be completely analyzed before $M$ and the result of analyzing $N$ is used in analyzing $M$. This is the behavior of Milner's algorithm $\mathcal{W}[21]$.

2. Because the only notion of principality requires fixing the part of the type environment containing polytypes, for an arbitrarily chosen HM-typable term there does not seem to be an HM typing which represents all possible typings for that term. Later in this paper it is shown in fact that this is not mere appearance - individual HM typings can not be used to represent all possible HM typings for a term. This makes it more difficult to use the HM type system for approaches that involve incremental or separate computation of type information. So HM may not be right for some applications.

\subsection{Principal Typings with Intersection Types}

At the present time, for a type system to support both type polymorphism and principal typings, the most promising approaches rely on the use of intersection types. There is not room in this paper to go into much detail about intersection types, so the discussion here gives only the highlights.

The first system of intersection types for which principal typings was proved was presented by Coppo, Dezani, and Venneri [3] (a later version is [4]). The same general approach has been followed by Ronchi Della Rocca and Venneri [30] and van Bakel [34] for other systems of intersection types. In this approach, finding a principal typing algorithm for a term $M$ involves

- finding a normal form (or approximate normal form) $M^{\prime}$ for $M$,

- assigning a typing $t$ to $M^{\prime}$,

- proving that any typing for the normal form $M^{\prime}$ is also a typing for the original term $M$, and

- proving that any other typing $t^{\prime}$ for the normal form $M^{\prime}$ can be obtained from $t$ by a sequence of operations each of which is one of expansion (sometimes called duplication), lifting (implementing subtyping, sometimes called rise), or substitution.

This general approach is summarized nicely in $[35, \S 5.3]$. This is intrinsically an impractical method and hence is primarily of theoretical interest. The definitions of the operations on typings are sometimes quite complicated, so they will not be discussed in this paper.

The first unification-based approach to principal typing with intersection types is by Ronchi Della Rocca [29]. An always-terminating restriction is pre- 
sented which bounds the height of types. Unfortunately, this approach uses a complicated approach to expansion and is thus quite difficult to understand.

The first potentially practical approaches to principal typing with intersection types were subsequent unification-based methods which focused on the rank-2 restriction of intersection types. Van Bakel presented a unification algorithm for principal typing for a rank-2 system [34]. Later independent work by Jim also attacks the same problem, but with more emphasis on handling practical programming language issues such as recursive definitions, separate compilation, and accurate error messages [15]. Successors to Jim's method include Banerjee's [1], which integrates flow analysis, and Jensen's [13], which integrates strictness analysis. Other approaches to principal typings and type inference with intersection types include [5] and [12].

The most recent development in this area is the introduction of the notion of expansion variables [19]. The key idea is that with expansion variables, the earlier notions of expansion and substitution can be integrated in a single notion of substitution called $\beta$-substitution. This results in a great simplification over earlier approaches beyond the rank-2 restriction. However, there are still many technical issues needing to be overcome before this is ready for general use.

\subsection{An Observation about all Previous Definitions}

The following holds for each system $S$ with principal typings that I know about. In $S$, each typable term $M$ can be assigned a typing $t$ which is principal for $M$ in the sense that for every other typing $t^{\prime}$ assignable to $M$, there exist operations $O_{1}, \ldots, O_{n}$ such that

$-t^{\prime}=O_{n}\left(\cdots\left(O_{1}(t)\right) \cdots\right)$, and

- the operations are sound in the sense that for any term $N$, if $S \triangleright N: t$, then $S \triangleright N: t_{i}$ where $t_{i}=O_{i}\left(\cdots\left(O_{1}(t)\right) \cdots\right)$ for $1 \leq i \leq n$.

For some (but not all) systems a stronger statement about the soundness of the operations holds:

For $0 \leq i<n$ and any term $N$, if $S \triangleright N: t^{\prime}$, then $S \triangleright N: O_{i}\left(t^{\prime}\right)$.

For STLC, these operations are substitution and weakening, which are sound in the stronger sense (provided weakening is defined to do nothing on typings already mentioning the term variable in question). For various systems with intersection types, these operations are expansion, lifting, rise, as well as substitution and weakening. In some systems with intersection types, these operations are sound in the stronger sense, and in others, they are sound in the weaker sense.

Observation 3 In each such system $S$, if $t$ is principal for $M$, then $t \leq_{S} t^{\prime}$ for every $t^{\prime} \in \operatorname{Typings}_{S}(M)$.

\section{A New Definition of Principal Typing}

In designing a general definition of "principal typing", the important issue seems to be the following: 
A principal typing in $S$ for a term $M$ should be a typing for $M$ which somehow represents all other possible typings in $S$ for $M$.

This paper has already introduced the new technical machinery necessary to capture this notion in the information order $\leq_{S}$ on typings. This suggests that the following new definition is the right one.

Definition 4 (Principal Typing). A typing $t$ is principal in system $S$ for term $M$ iff $S \triangleright M: t$ and $S \triangleright M: t^{\prime}$ implies $t \leq_{S} t^{\prime}$.

\subsection{Positive Results}

The first thing to check about the new definition is whether existing definitions for specific type systems are instances of the new definition. In all cases, every typing that is principal by one of the old definitions is principal by the new one. This is justified by observation 3 .

It remains to be considered whether every typing in a system $S$ that is principal by the new definition is also principal by the old one for $S$. For STLC, the new definition corresponds exactly to the old definition.

Theorem 4. A typing $t$ is principal in STLC for $M$ according to definition 2 iff $t$ is principal in STLC for $M$ according to definition 4.

All proofs are in the appendix.

For some other type systems, the new definition will be slightly more liberal, accepting some additional typings as being principal. For example, consider the term $\omega=(\lambda x . x x)$. The usual principal typing of $\omega$ in a system with intersection types is $t_{1}=(\emptyset \vdash(\alpha \rightarrow \beta) \cap \alpha \rightarrow \beta)$. With the new definition, $t_{2}=(\emptyset \vdash$ $(\alpha \rightarrow \beta) \cap \alpha \cap \gamma \rightarrow \beta)$ is also a principal typing, because in some systems with intersection types a term can be assigned $t_{1}$ iff it can be assigned $t_{2}$. This is merely a harmless quirk. The old definitions ruled out unneeded intersections with type variables because they were inconvenient.

\subsection{Type Systems without Principal Typings}

The new definition 4 of principal typings can be used to finally prove that certain type systems do not have principal typings. These results are significant, as clarified by this statement by Jim in [14] about the best previous knowledge:

"This imprecision [in the definition of principal typings] makes it impossible for us to prove that a given type system lacks the principal type property."

Theorem 5. The HM system does not have principal typings for all terms.

It is quite important that the research community is made aware of Theorem 5, because as Jim points out in [14], "a number of authors have published offhand claims that ML possesses the principal typings property".

Theorem 6. System $\mathrm{F}$ does not have principal typings for all terms. 


\section{References}

[1] A. Banerjee. A modular, polyvariant, and type-based closure analysis. In Proc. 1997 Int'l Conf. Functional Programming. ACM Press, 1997.

[2] H. P. Barendregt. The Lambda Calculus: Its Syntax and Semantics. North-Holland, revised edition, 1984

[3] M. Coppo, M. Dezani-Ciancaglini, B. Venneri. Principal type schemes and $\lambda$-calculus semantics. In Hindley and Seldin [11].

[4] M. Coppo, M. Dezani-Ciancaglini, B. Venneri. Functional characters of solvable terms. Z. Math. Logik Grundlag. Math., 27(1), 1981.

[5] M. Coppo, P. Giannini. A complete type inference algorithm for simple intersection types. In 17th Colloq. Trees in Algebra and Programming, vol. 581 of LNCS. Springer-Verlag, 1992.

[6] H. B. Curry, R. Feys. Combinatory Logic I. Studies in Logic and the Foundations of Mathematics. North-Holland, Amsterdam, 1958.

[7] L. Damas, R. Milner. Principal type schemes for functional programs. In Conf. Rec. 9th Ann. ACM Symp. Princ. of Prog. Langs., 1982

[8] J.-Y. Girard. Interprétation Fonctionnelle et Elimination des Coupures de l'Arithmétique d'Ordre Supérieur. Thèse d'Etat, Université de Paris VII, 1972.

[9] R. Harper, J. C. Mitchell. On the type structure of Standard ML. ACM Trans. on Prog. Langs. ES Systs., 15(2), 1993.

[10] J. R. Hindley. Basic Simple Type Theory, vol. 42 of Cambridge Tracts in Theoretical Computer Science. Cambridge University Press, 1997.

[11] J. R. Hindley, J. P. Seldin, eds. To H. B. Curry: Essays on Combinatory Logic, Lambda Calculus, and Formalism. Academic Press, 1980.

[12] B. Jacobs, I. Margaria, M. Zacchi. Filter models with polymorphic types. Theoret. Comp. Sci., 95, 1992.

[13] T. Jensen. Inference of polymorphic and conditional strictness properties. In POPL '98 [26].

[14] T. Jim. What are principal typings and what are they good for? Tech. memo. MIT/LCS/TM532, MIT, 1995.

[15] T. Jim. What are principal typings and what are they good for? In Conf. Rec. POPL '96: 23rd ACM Symp. Princ. of Prog. Langs., 1996.

[16] P. Jouvelot, D. K. Gifford. Algebraic reconstruction of types and effects. In Conf. Rec. 18th Ann. ACM Symp. Princ. of Prog. Langs., 1991.

[17] A. J. Kfoury, H. G. Mairson, F. A. Turbak, J. B. Wells. Relating typability and expressibility in finite-rank intersection type systems. In Proc. 1999 Int'l Conf. Functional Programming. ACM Press, 1999

[18] A. J. Kfoury, J. B. Wells. A direct algorithm for type inference in the rank-2 fragment of the second-order $\lambda$-calculus. In Proc. 1994 ACM Conf. LISP Funct. Program., 1994.

[19] A. J. Kfoury, J. B. Wells. Principality and decidable type inference for finite-rank intersection types. In Conf. Rec. POPL '99: 26th ACM Symp. Princ. of Prog. Langs., 1999.

[20] D. Leivant. Polymorphic type inference. In Conf. Rec. 10th Ann. ACM Symp. Princ. of Prog. Langs., 1983.

[21] R. Milner. A theory of type polymorphism in programming. J. Comput. System Sci., 17, 1978 [22] R. Milner, M. Tofte, R. Harper, D. B. MacQueen. The Definition of Standard ML (Revised). MIT Press, 1997.

[23] J. C. Mitchell. Polymorphic type inference and containment. In G. Huet, ed., Logical Foundations of Functional Programming, chapter 8. Addison-Wesley, 1990

[24] J. H. Morris. Lambda-calculus Models of Programming Languages. PhD thesis, Massachusetts Institute of Technology, Cambridge, Mass., U.S.A., 1968.

[25] B. Pierce. Bounded quantification is undecidable. Inform. \&6 Comput., 112, 1994

[26] Conf. Rec. POPL '98: 25th ACM Symp. Princ. of Prog. Langs., 1998.

27 G. Pottinger. A type assignment for the strongly normalizable $\lambda$-terms. In Hindley and Seldin [11]

[28] J. C. Reynolds. Towards a theory of type structure. In Colloque sur la Programmation, vol. 19 of $L N C S$, Paris, France, 1974. Springer-Verlag.

[29] S. Ronchi Della Rocca. Principal type schemes and unification for intersection type discipline. Theoret. Comp. Sci., 59(1-2), 1988.

[30] S. Ronchi Della Rocca, B. Venneri. Principal type schemes for an extended type theory. Theoret. Comp. Sci., 28(1-2), 1984.

[31] A. Schubert. Second-order unification and type inference for Church-style polymorphism. In POPL '98 [26]

[32] J.-P. Talpin, P. Jouvelot. The type and effect discipline. Inform. E Comput., 111(2), 1994.

33] P. Urzyczyn. Type reconstruction in $\mathbf{F}_{\omega}$. Math. Structures Comput. Sci., 7(4), 1997.

[34] S. J. van Bakel. Intersection Type Disciplines in Lambda Calculus and Applicative Term Rewriting Systems. PhD thesis, Catholic University of Nijmegen, 1993.

[35] S. J. van Bakel. Intersection type assignment systems. Theoret. Comp. Sci., 151(2), 1995.

[36] J. B. Wells. Typability and type checking in the second-order $\lambda$-calculus are equivalent and undecidable. In Proc. 9th Ann. IEEE Symp. Logic in Comp. Sci., 1994. Superseded by [39]. 
[37] J. B. Wells. Typability is undecidable for F+eta. Tech. Rep. 96-022, Comp. Sci. Dept., Boston Univ., 1996.

[38] J. B. Wells. Type Inference for System F with and without the Eta Rule. Ph.D. thesis, Boston Univ., 1996.

[39] J. B. Wells. Typability and type checking in System F are equivalent and undecidable. Ann. Pure Appl. Logic, 98(1-3), 1999. Supersedes [36].

\section{Appendix: Proofs}

\subsection{Properties of STLC}

The analysis of the new definition vs. the old definition for STLC proceeds in two parts.

Lemma 1. Let $t=(A \vdash \tau)$ be a STLC typing (maybe or maybe not derivable).

1. Then $t \leq \mathrm{STLC} s(t)$ for any type substitution $s$.

2. Then $t \leq \mathrm{STLC}\left(A \cup A^{\prime} \vdash \tau\right)$ for any type environment $A^{\prime}$ such that $\operatorname{dom}(A) \cap$ $\operatorname{dom}\left(A^{\prime}\right)=\emptyset$.

Proof.

1. By $[10, \S 3 \mathrm{~A} 2.1(\mathrm{ii})]$, if STLC $\triangleright M: t$, then STLC $\triangleright M: s(t)$.

2. By $[10, \S 2 \mathrm{~A} 9.1]$, if STLC $\triangleright M:(A \vdash \tau)$, then STLC $\triangleright M:\left(A \cap A^{\prime} \vdash \tau\right)$.

Theorem 7. Let $t$ be principal in STLC for $M$ according to definition 2. Then $t$ is principal in STLC for $M$ according to definition 4.

Proof. Let $t=(A \vdash \tau)$.

By definition 2, STLC $\triangleright M: t$.

Suppose $S \triangleright M: t^{\prime}$ for some typing $t^{\prime}=\left(A^{\prime} \vdash \tau^{\prime}\right)$. By definition 2 , there exists some $s$ such that $A^{\prime} \supseteq s(A)$ and $\tau^{\prime}=s(\tau)$. By lemma $1(1), t \leq$ sTLC $(s(A) \vdash s(\tau))=\left(s(A) \vdash \tau^{\prime}\right)$. By lemma 1(2), $\left(s(A) \vdash \tau^{\prime}\right) \leq$ STLC $\left(A^{\prime} \vdash \tau^{\prime}\right)=t^{\prime}$. Thus, $t \leq$ STLC $t^{\prime}$.

Theorem 7 shows that the new definition of principal typing is at least as liberal as the old one for STLC. An even stronger statement is possible, because in the case of STLC the new and old definitions of principal typing match exactly.

Lemma 2. Let $t$ be principal in STLC for $M$ according to definition 2. Let $t^{\prime}$ be a typing in STLC for $M$ which is not principal according to definition 2. Then $t^{\prime} \nless$ STLC $t$.

Proof. Let $t=(A \vdash \tau)$ and let $t^{\prime}=\left(A^{\prime} \vdash \tau^{\prime}\right)$. By definition 2, STLC $\triangleright M: t$ and there exists some $s$ such that $A^{\prime} \supseteq s(A)$ and $\tau^{\prime}=s(\tau)$. Because $t^{\prime}$ is not principal, there does not exist $s^{\prime}$ such that $A \supseteq s^{\prime}\left(A^{\prime}\right)$ and $\tau=s^{\prime}\left(\tau^{\prime}\right)$.

1. Suppose $A^{\prime} \neq s(A)$. Let $x \in \operatorname{dom}\left(A^{\prime}\right) \backslash \operatorname{dom}(A)$. Let $y \notin \mathrm{FV}(M)$. Let $N=$ $((\lambda y M) x)$ 
2. Suppose otherwise, i.e., $A^{\prime}=s(A)$. Define the function $\Phi$ as follows:

$$
\begin{aligned}
& \Phi(\alpha, \alpha)=(\lambda y w \cdot w(y z)(y x)) \\
& \Phi(\sigma \rightarrow \tau, \alpha)=(\lambda y w \cdot w(x y)((\lambda x \cdot \Phi(\sigma, \alpha)) y)) \\
& \text { if } \alpha \in \operatorname{FTV}(\sigma) \\
& \Phi(\sigma \rightarrow \tau, \alpha)=(\lambda w \cdot(\lambda x . \Phi(\tau, \alpha))(x w)) \\
& \quad \text { otherwise. }
\end{aligned}
$$

(a) Suppose $s(\alpha)$ is not a type variable for $\alpha \in \operatorname{FTV}(t)$.

i. Suppose $\alpha \in \operatorname{FTV}(\tau)$. Let $N=(\lambda x .(\lambda u . x)(\lambda w .(\lambda z \cdot \Phi(\tau, \alpha))(\lambda v \cdot w))) M$.

ii. Suppose $\alpha \in \operatorname{FTV}(A(w))$ for some $w \in \operatorname{FV}(M)$. Let $y \notin \mathrm{FV}(M)$. Let $N=(\lambda y \cdot M)(\lambda u .(\lambda x z . \Phi(A(z), \alpha)) w(\lambda v \cdot u))$.

(b) Suppose $s\left(\alpha_{1}\right)=s\left(\alpha_{2}\right)=\beta$ for distinct $\alpha_{1}, \alpha_{2} \in \mathrm{FTV}(t)$. W/o.l.o.g., assume that $\alpha_{i} \in \operatorname{FTV}\left(A\left(w_{i}\right)\right)$ where $w_{i} \in \mathrm{FV}(M)$ for $i \in\{1,2\}$. Let $P_{i}=\left(\left(\lambda x . \Phi\left(A\left(w_{i}\right), \alpha_{i}\right)\right) w_{i}\right)$ for $i \in\{1,2\}$. Let $P=\left(\lambda z u . u P_{1} P_{2}\right)$. Let $N=(\lambda x y \cdot x) M P$.

Then $N \in \operatorname{TermssTLC}\left(t^{\prime}\right) \backslash \operatorname{TermsSTLC}(t)$. Thus, $t^{\prime} \not \mathbf{S T L C} t$.

Proof of Theorem 4 .

$\Rightarrow$ By theorem 7 .

$\Leftarrow$ Suppose $t$ is not principal in STLC for $M$ according to definition 2. By lemma 2, it follows that $t$ is not principal in STLC for $M$ according to definition 4.

\subsection{Properties of HM}

Proof of Theorem 5. It is sufficient to find one term without a principal typing. The term $(x x)$ will be proven not to have a principal typing. Every typing of $(x x)$ will be proven not to be principal.

Let $(A \vdash \tau)$ be any typing assignable to $(x x)$ in HM. Then HM $\triangleright(x x):(A \vdash \tau)$.

It will now be proven that $(A \vdash \tau)$ is not principal. The strategy is to find $A^{\prime}, t^{\prime}$, and $M$ such that:

$$
\begin{aligned}
& \mathrm{HM} \triangleright(x x):\left(A^{\prime} \vdash \tau^{\prime}\right) \\
& \mathrm{HM} \triangleright M:(A \vdash \tau) \\
& \mathrm{HM} \triangleright M:\left(A^{\prime} \vdash \tau^{\prime}\right)
\end{aligned}
$$

Let $\left(\forall \vec{\alpha} \cdot \tau^{\prime \prime}\right)=A(x)$ where $\tau^{\prime \prime}$ is a monotype. Let $k$ be the length of the leftmost path in $\tau^{\prime \prime}$ (viewed as a tree). Observe that the leaf at the end of this path must be $\alpha_{i}$ for $1 \leq i \leq n$.

Let $\sigma^{\prime}=\left(\forall \cdot \tau^{\prime \prime} \rightarrow \beta\right)$, where $\beta$ is a fresh type variable. (The notation $(\forall \cdot \tau)$ is shorthand for quantifying over all free type variables in $\tau$.)

Let $A^{\prime}=\left\{x: \sigma^{\prime}\right\}$. Let $\tau^{\prime}$ be any arbitrarily chosen type.

It is clear that $\mathrm{HM} \triangleright(x x):\left(A^{\prime} \vdash \tau^{\prime}\right)$.

Let $K=(\lambda x y \cdot x)$.

Let $N_{0}=\mathrm{c}$.

Let $\operatorname{Unify}\left(N, N^{\prime}\right)=\left(\lambda x y . y(x N)\left(x N^{\prime}\right)\right)$ where $x$ and $y$ are fresh. 
Let $N_{i+1}=\left(\lambda x . K y_{i+1} \operatorname{Unify}\left(x, N_{i}\right)\right)$ where $x, y_{1}, \ldots, y_{i+1}$ are distinct.

Observe that the derived type of $N_{i}$ will have a leftmost path of length $i$ and a ground type at the end of the path.

Let $M=K(x x)\left(\lambda y_{1} \ldots y_{k} . x N_{k}\right)$.

It is not hard to check that HM $\triangleright M:(A \vdash \tau)$.

It is impossible to derive $M:\left(A^{\prime} \vdash \tau^{\prime}\right)$ in $\mathrm{HM}$ because the leftmost branch in $\sigma^{\prime}$ is too big, so there is no instantiation of $\sigma^{\prime}$ which allows typing the subterm $\left(x N_{k}\right)$.

Therefore, $(A \vdash \tau)$ is not a principal typing for $(x x)$.

\subsection{Properties of $F$}

Proof of Theorem 6. It is sufficient to find one term without a principal typing. The term $(x x)$ will be proven not to have a principal typing. Every typing of $(x x)$ will be proven not to be principal.

The strategy is to suppose that $t=(A \vdash \tau)$ is a principal typing for the term $(x x)$ and then to derive a contradiction, thereby proving that $(x x)$ has no principal typing. Let $\sigma^{\prime}$ be the type resulting from erasing all occurrences of " $\forall$ " from the type $\sigma=A(x)$ and replacing all formerly bound type variables by fresh, distinct, free type variables. Let $B$ be a new set of type assumptions such that $B(x)=\left(\forall \cdot\left(\sigma^{\prime} \rightarrow \beta\right)\right)$ where $\beta$ is some freshly chosen type variable. (The notation $\forall . \tau$ is shorthand for quantifying over all free type variables in $\tau$.) Let $t^{\prime}$ be the typing $(B \vdash \rho)$ for some arbitrarily chosen type $\rho$. Using the supposed fact that $\mathrm{F} \triangleright(x x): t$, it is not hard to check that $\mathrm{F} \triangleright(x x): t^{\prime}$. Then the methods of $[36,37,38,39]$ can be used to build a program fragment $M$ such that $((\lambda y . x x) M) \in \operatorname{Terms}_{\mathrm{F}}(t) \backslash \operatorname{Terms}_{\mathrm{F}}\left(t^{\prime}\right)$, contradicting the claim that $t$ is principal in $\mathrm{F}$ for $(x x)$. The fragment $M$ in a sense "tests" the size of the type of $x$ and fails if the type is too big, which it is in $t^{\prime}$ but not in $t$. Specifically, $M$ is of the form $C[y x]$ where $C$ captures $y$ but not $x$. The variable $y$ is forced by the shape of $C$ to have the type $\forall .(\sigma \rightarrow \alpha)$ for some fresh type variable $\alpha$. Because of embedded $\forall$ quantifiers at the root of $\sigma$, this type can not "stretch" to be as big as $\left(\sigma^{\prime} \rightarrow \beta\right) \rightarrow \alpha$, so the application $(y x)$ can not be typed in $t^{\prime}$. 